\title{
EDITORIAL
}

\section{The decision to donate: helping families make meaning during neonatal loss. In response to: anencephalic organ donation after cardiac death: practicalities and ethics - a case report}

\section{Journal of Perinatology (2015) 35, 777-778. doi:10.1038/jp.2015.101}

Every day in the United States, 21 people die on the organ waitlist. ${ }^{1}$ As clinicians, researchers and institutions seek additional opportunities to expand organ and tissue donation, we must continue to learn from the ethical, emotional and psychological implications of donation opportunities for potential donors and their families. The article in this issue by Powers et al. describes the experience of meeting one family's request that their infant daughter with anencephaly, 'Baby Amelia,' be considered as a potential organ or tissue donor. The authors note that permitting donation after circulatory death (DCD) by infants with anencephaly offers the possibility for the transplantation of organs that were previously considered non-transplantable and may result in more lives saved. We suggest that an equally important outcome is the sense of meaning and healing that many families experience with a decision to consider organ or tissue donation for their child.

Although it is possible that the clinical course for infants with anencephaly may be more likely to preclude successful organ or tissue retrieval, as it did for Baby Amelia, finding meaning in the donation process is not contingent on a successful transplant. Hogan et al. $^{2}$ found that families of tissue donors report several important aspects of meaning-making, including doing the right thing, helping others and believing something good came from the death. Data suggest that donation has a positive impact on the grief process for many families. ${ }^{3}$ Grieving individuals and donor families who feel they can make meaning and sense out of their loved one's death are less likely to show symptoms of complex and prolonged grief, specifically in the case of child loss. ${ }^{4}$ Many parents of pediatric donors specifically experienced a sense of meaning and comfort knowing their decision could prevent another parent from feeling the same devastating loss they are experiencing.

Some clinicians may worry that families are not interested in learning about organ donation in the case of neonatal loss, though several studies suggest that families often request this information. Labrecque et al. ${ }^{5}$ describe the results of a medical record review performed at several Boston area hospitals for neonatal deaths, including that a significant number of medical records indicated families' interest in potential organ donation. These results echo those of Naim et al. ${ }^{6}$ who found that, in $75 \%$ of patients identified as potential DCD donors, the first mention of donation was initiated by the family. Clinicians with first-hand experience of the pediatric organ donation process confirm that organ donation helps families during the grieving process. ${ }^{7}$

Unfortunately, as described in the case report, Baby Amelia's organs were ultimately unable to be recovered and transplanted based on the length of time she survived following the removal of mechanical ventilation. As the authors point out, 'the DCD approach is based on the assumption that the patient is dependent on life support and will expire shortly after such support is removed. However, the deteriorating clinical course of anencephalic infants is variable and unpredictable because the specific time course of the terminal process is related to the degree of dysfunction of the brainstem.' Though actual success rates for anencephalic donation are unknown at this time, the difficulty predicting whether death will occur in the necessary time frame for donation to occur suggests cases like that of Baby Amelia may not be uncommon as anencephalic DCD donation becomes more prevalent.

We must then remain vigilant to the potential for a sense of secondary loss for parents in circumstances of anencephalic DCD donation. In any scenario, there are no guarantees of outcome following the decision to donate. There is always the possibility that organs or tissues will not be recovered or transplanted, that they may be rejected after transplant, or that the recipient will die despite the transplant. There is a dearth of research regarding the emotional and psychological impact on families who have authorized donation for a loved one who ultimately becomes ineligible to donate owing to medical circumstances. In their 2011 article on secondary loss in donor families, Corr et al. ${ }^{3}$ present some of the only information available on this topic, analyzing three specific case examples. They found that an individual who learns that their loved one's organs or tissues were unable to be utilized because of medical or social contraindications may experience a secondary grief reaction, similar to the reaction felt about the death itself.

As anencephalic DCD is explored at Organ Procurement Organizations and hospitals, we must ensure that families have a clear understanding of the DCD donation process and a realistic understanding of all possible outcomes of donation, including successful transplant or an inability to recover or transplant for medical reasons. ${ }^{3}$ Ensuring the participation of clinical social workers or psychologists in facilitating donation discussions provides a higher level of emotional and psychological support to families throughout the complex decision-making and clinical process steps. A key responsibility of the family support team is to reinforce for families the value in the donation decision itself. As Chatziioannidis et al. ${ }^{8}$ suggest in their article exploring newborn donation in Europe, 'Open and honest information in order to reduce parent's sense of loss of control due to their newborn's vulnerability and inability to claim their rights should be the primary policy for transplantation networks.'

Calls for development of neonatal DCD donation protocols recognize that the decision to donate can be a meaningful part of grief recovery for families.9 Such protocols must include provisions for honor, recognition and aftercare for cases like that of Baby Amelia. Through the growth of 'donor in spirit' programs, Organ Procurement Organizations, hospitals and transplant centers are able to ensure that donors whose gifts do not result in physical transplants are honored and recognized in the same way as donors whose organs are transplanted. These 'donors in spirit' programs often include donor remembrance events, engraved donor memorial walls, raising donor flags in honor of the donor in spirit and tangible mementos for families to keep. Including 'donor in spirit' programs as required followup care for any anencephalic DCD case that does not result in a transplant can help to mitigate any secondary loss, focusing 
instead on the meaning of the decision to donate rather than the outcome. We suggest this type of aftercare programming be required for any institution developing anencephalic DCD donation protocols.

Organ donation programs throughout the United States adhere to high standards of training and competency associated with the support and care of donor families. As outlined in the Association of Organ Procurement Organization's (AOPO) Standards, which are required for accreditation, programs offer all organ donor families comprehensive services. ${ }^{10}$ The AOPO program also outlines standards for DCD clinical processes, which complements the guidelines, policies and best practices promulgated by the United Network for Organ Sharing and the Centers for Medicare and Medicaid Services. These standards and best practices currently serve the families of all donors and can support the development of programs that target the clinical and family support needs of potential pediatric and neonate donors. These standards offer frameworks for meeting the needs of families like that of Baby Amelia, whose loved ones offer the gift of hope as a 'donor in spirit'.

\section{CONFLICT OF INTEREST}

As employees of The Living Legacy Foundation of Maryland, an Organ Procurement Organization, L Williams and K Kennedy have potential conflict of interest. RD Boss declares no conflict of interest.

$\mathrm{L}_{\text {Williams }}{ }^{1}, \mathrm{~K}$ Kennedy ${ }^{1}$ and RD Boss ${ }^{2}$

${ }^{1}$ The Living Legacy Foundation of Maryland, Baltimore, MD, USA and
${ }^{2}$ Division of Neonatology, Department of Pediatrics, Johns Hopkins University School of Medicine, Berman Institute of Bioethics,

Baltimore, MD, USA

E-mail: rboss1@jhmi.edu

\section{REFERENCES}

1 U.S. Department of Health and Human Services, Organ Procurement and Transplantion Network. http://optn.transplant.hrsa.gov/, 2015. Accessed 4 June 2015.

2 Hogan NS, Coolican M, Schmidt LA. Making meaning in the legacy of tissue donation for donor families. Prog Transplant 2013; 23(2): 180-187.

3 Corr CA, Coolican MB, Moretti LS, Simon R. Donor families, distinctive secondary losses, and "second death" experiences. Prog Transplant 2011; 21(3): 220-227.

4 Keesee NJ, Currier JM, Neimeyer RA. Predictors of grief following the death of one's child: the contribution of finding meaning. J Clin Psychol 2008; 64(10): 1145-1163.

5 Labrecque M, Parad R, Gupta M, Hansen A. Donation after cardiac death: the potential contribution of an infant organ donor population. $J$ Pediatr 2011; 158(1): 31-36.

6 Naim MY, Hoehn KS, Hasz RD, White LS, Helfaer MA, Nelson RM. The Children's Hospital of Philadelphia's experience with donation after cardiac death. Crit Care Med 2008; 36(6): 1729-1733.

7 Mathur M, Taylor S, Tiras K, Wilson M, Abd-Allah S. Pediatric critical care nurses' perceptions, knowledge, and attitudes regarding organ donation after cardiac death. Pediatr Crit Care Med 2008; 9(3): 261-269.

8 Chatziioannidis I, Chouchou P, Mitsiakos G. Newborns as potential organ donors: a new perspective? J Pediatr Neonat Individual Med 2015; 4(1): e040114.

9 Himelstein BP, Hilden JM, Boldt AM, Weissman D. Pediatric palliative care. N Engl J Med 2004; 350(17): 1752-1762.

10 AOPO Standards and Interpretive Guidelines. http://www.aopo.org/, 2015 Accessed 4 June 2015. 